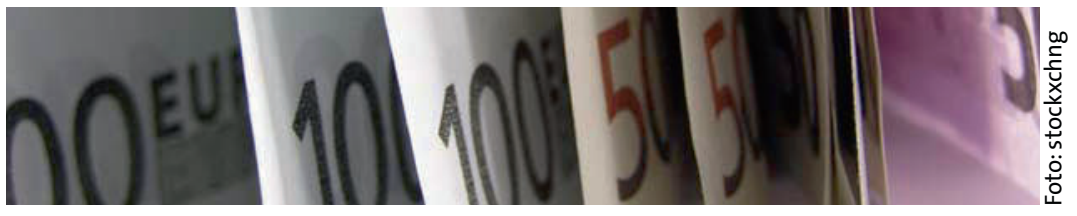

\title{
Wie viel Allergologie können wir uns noch leisten?
}

\author{
Pharmazeutische Innovationen kosten. Können wir uns das in der \\ Allergologie noch leisten? Wollen wir uns das überhaupt noch leisten \\ oder sind manche Produkte einfach zu teuer? Auch solche brisanten \\ Fragen wurden beim Allergiekongresses in Berlin diskutiert.
}

\begin{abstract}
A llergie kostet - auch die Volkswirtschaft, wenn allergische Erkrankungen nicht ausreichend behandelt werden, betonte Prof. Dr. Torsten Zuberbier aus Berlin. So beeinträchtigen allergische Erkrankungen messbar die Leistungsfähigkeit und sind gerade bei Menschen in Schule und Beruf besonders häufig. Doch die weitgehend fehlende Kostenübernahme für die symptomatische Therapie allergischer Erkrankungen erschwert die Versorgung der betroffenen Patienten. Ebenso ist in der Vergütung allergologischer Leistungen der Wurm drin: Während Hautärzte pro Quartal bei einer Durchschnittsfallzahl von 1.286 Fällen nach dem Regelleistungsvolumen einen Fallwert von $17,36 €$ zu beklagen haben, rechnen Heilpraktiker für eine eingehende, mindestens zehnminütige Beratung bis zu $22 €$ ab, erregte sich Zuberbier. Das zeige aber auch eine gesellschaftliche Perspektive: Menschen sind bereit, für nicht gesicherte Diagnosen und Therapien tief in die Tasche zu greifen.
\end{abstract}

\section{Auto kommt vor Gesundheit}

Die Ausgaben der gesetzlichen Krankenkassen für gesicherte medizinische Leistungen erscheinen da gar nicht so üppig: Der Verband der Angestelltenkrankenkassen hat für das Jahr 2006 berechnet, dass die Gesamtausgaben pro Versicherten und Monat bei ca. 170,80€ liegen. Ein Auto kostet mehr: Die Haltung eines VW-Golfs schlägt im Monat beispielsweise mit $556 € \mathrm{zu}$ Buche, rechnete $\mathrm{Zu}$ berbier vor. Wir müssen als Gesellschaft entscheiden, was wichtiger ist.
Darin stimmten auch Ch.-Markos Dintsios vom Institut für Qualität und Wirtschaftlichkeit im Gesundheitswesen (IQWiG), Bonn, und Tobias Gantner von Novartis Pharma Deutschland, Nürnberg, mit Zuberbier überein. Die finanziellen Mittel im Gesundheitswesen seien nun einmal endlich und zudem noch konjunkturabhängig. Die Gesundheitsausgaben haben sich in Deutschland alleine von 1970 bis 2002 fast verdoppelt auf $11,2 \%$ des Bruttoinlandsprodukts. Dabei ist die Zahl der Verordnungen dramatisch gesunken, während der Arzneimittelumsatz deutlich zugenommen hat, wie Dintsios aus dem Arzneimittelreport zitierte. Dahinter stehen vor allem kostspielige Neuerungen. Allerdings gebe es auch noch viel Potenzial zur Rationalisierung ohne Rationierung. Dintsios nannte hier insbesondere die im internationalen Vergleich besondere $\mathrm{Si}$ tuation von immer noch sehr vielen Altzulassungen hierzulande. In anderen Ländern gäbe es Negativlisten, die solche Substanzen vom Markt verdrängten.

\section{Teures Deutschland}

Innovationen sind kostspielig. Im Detail lässt sich die Industrie nicht in die Karten schauen, es kursieren aber Zahlen aus dem Jahr 2001, die die Kosten pro Neueinführung mit 800 Millionen US-\$ beziffern, wie Zuberbier berichtete. Für ein neues Allergoid fallen laut Gantner reine Entwicklungskosten von 13,5 Millionen Euro an. Bei einer Entwicklungszeit von bis zu zwölf Jahren ist die Zeit zur Nutzung des Patents, also die Amortisie- rungsphase, kurz - möglicherweise wäre eine Verlängerung hilfreich, um zu moderateren Preisen zu kommen.

Die Arzneimittelpreise sind in Deutschland insgesamt immer noch vergleichsweise hoch. International sind auch willkürliche Preisfestlegungen und Preiskürzungen bei Arzneimitteln möglich, berichtete Dintsios. So werden in manchen Ländern zur Preisfindung internationale Vergleichswerte herangezogen (Kanada, Italien, Niederlande, Schweiz) oder die Unternehmen unterstehen direkt einer Preiskontrolle (Großbritannien) oder es wird eine am Nutzen orientierte Preisgestaltung versucht (Australien). In Deutschland sind letztlich die Herstellerabgabepreise unreguliert und können von den Pharmaunternehmen frei festgesetzt werden. Die Erstattungsbeträge können allerdings über Festbetragsgruppen reguliert werden und auch außerhalb dieser Gruppen ist eine Höchstbetragsregelung möglich, die ggf. Entwicklungskosten berücksichtigt und auf KostenNutzen-Analysen beruhen soll. Rabattverträge wirken derzeit zusätzlich auf deutsche Arzneimittelpreise ein.

\section{Kostenfaktor Biologika}

Preissteuerungsinstrumente greifen bisher noch kaum bei biotechnologischen Arzneimitteln, die bei immunologischen und onkologischen Erkrankungen eine immer größere Rolle spielen. Im Jahr 2007 machten laut Dintsios solche Spezialpräparate bereits $24,2 \%$ der Arzneimittelausgaben der gesetzlichen Krankenversicherung aus. 2008 verursachten alleine zehn neue Arzneimittel ein Kostenpotenzial von 12 Milliarden Euro. Bei einer Innovation wie dem Anti-IgEAntikörper Omalizumab fallen aktuell bei hoher Dosierung beispielsweise Jahrestherapiekosten von bis zu $31.720 €$ an. Dazu kommen noch $825 €$ für ein Kombinationspräparat mit Glukokortkoid und lang wirksamem $\beta$-Mimetikum. Solche Kosten müssen einem entsprechenden Nutzen gegenüberstehen, der auch etwaige unerwünschte Wirkungen berücksichtigt.

Symposium 12. Wie viel allergologische Innovation können und wollen wir uns leisten? 